\title{
Effect of Non-Passive Operator on Enhanced Wave-Based Teleoperator for Robotic-Assisted Surgery: First Case Study
}

Jing Guo*, Chao Liu, Philippe Poignet

Department of Robotics, LIRMM Montpellier, France

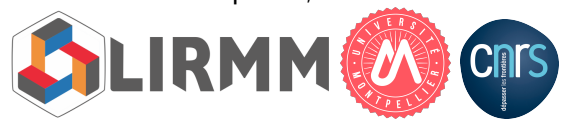

MesRob2015, Nantes, France - 9, July, 2015 


\section{Outline}

(1) Motivation

(2) Enhanced Wave Variable Architecture

- Fundamentals of Wave Variable Teleoperation

- Enhanced Wave Variable Teleoperation Structure

- Effect of Non-Passive Operator on Enhanced Wave Variable Structure

(3) Conclusions and Perspectives 
- Minimally invasive surgery (MIS) has advanced the surgical procedures in past decades.

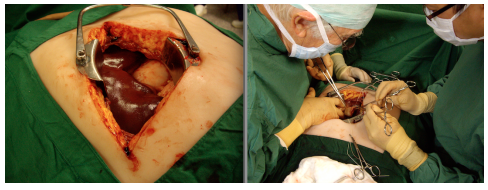

(a) Abdominal cavity surgery

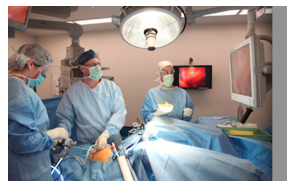

(b) Laparoscopic Surgery

Figure 1 : From open surgery to MIS ${ }^{1}$

Advantages of MIS: less invasiveness; less blood; shorten recovery time; reduced post-operative pain.

1 Fig1(a) is from Wellcome Trust 2011, UK; Fig1(b) is from Univeristy of MD SJMC, USA \& Greenslopes Specialist Gynaecology, Australia. 
- Miniaturized surgical robotic system presents promising trend for reducing invasiveness during surgical procedures .
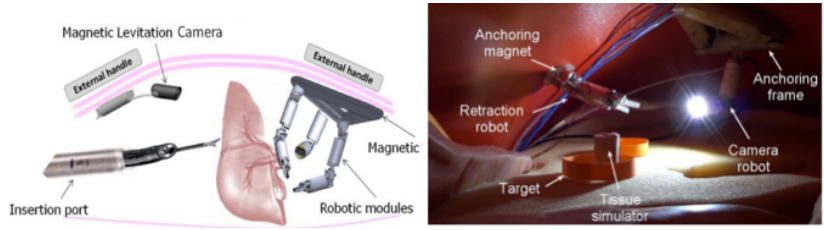

Figure 2: Modular Magnetic platform for Natural Orifice Translyminal Endoscopic Surgery [G.Tortora, 2013]

- However, cables for communication and power supply may affect the performance of system. 
- Miniaturized surgical robotic system presents promising trend for reducing invasiveness during operation.
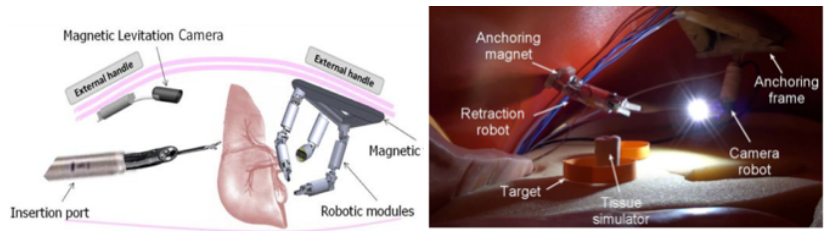

Wireless communication can replace cables for communication.

- But time delay will be introduced by wireless communication, thus induces stability issues for bilateral teleoperation system. 
Two criteria for bilateral teleoperation system:

- Stability - maintains stable (Safety);

- Transpareny - faithful transmission (tele-presence);

It is proved that stability and transparency are conflicting design goals in teleoperation system [D. Lawrence, 1993].

\section{Objectives}

1. Guarantee the stability of bilateral teleoperation system with time delay.

2. Improve transparency of bilateral teleoperation system with time delay. 


\section{0}

0000

0000000000

Fundamentals of Wave Variable Teleoperation

\section{Outline}

(1) Motivation

(2) Enhanced Wave Variable Architecture

- Fundamentals of Wave Variable Teleoperation

- Enhanced Wave Variable Teleoperation Structure

- Effect of Non-Passive Operator on Enhanced Wave Variable Structure

(3) Conclusions and Perspectives 
Standard Bilateral Teleoperation Model

- Standard bilateral teleoperation system normally consists five subsystems: human, master, communication, slave, and environment.

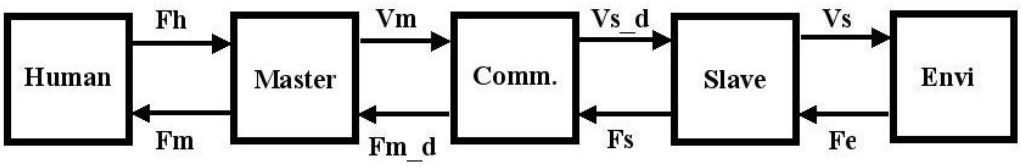

Figure 3: Standard Bilateral Teleoperation Model

- Velocities and force information are exchanged;

- Operator, master, slave and environment are assumed to be passive; 
Standard Bilateral Teleoperation Model

\section{Scattering theory}

A system is passive if and only if the norm of its scattering operator $S$ is less than or equal to one: $\|S(s)\| \leq 1$

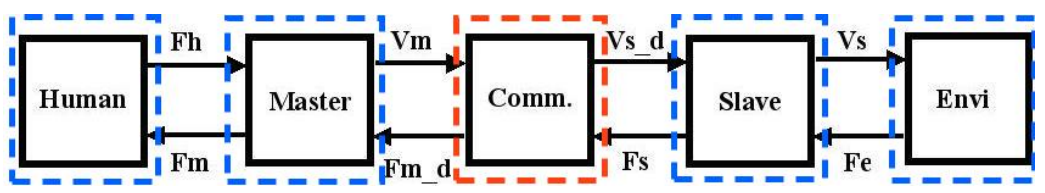

Figure 4: Standard Bilateral Teleoperation Model

- Analysis the time delay through scattering theory: $\|S(s)\|=\infty$

- Direct transmission of force and velocity signal with time delay is not passive. 


\section{0}

0000

0000000000

Fundamentals of Wave Variable Teleoperation

Wave Variable Based Teleoperation Method

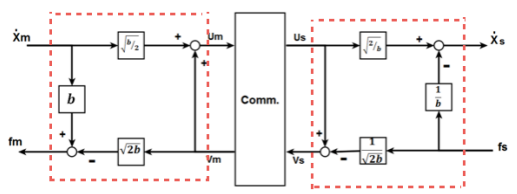

Figure 5: Wave variable based teleoperation scheme Outgoing wave variables $u_{m}, v_{s}$ are constructed as:

$u_{m}(t)=\frac{1}{\sqrt{2 b}}\left(f_{m}(t)+b \dot{x}_{m}(t)\right) \quad v_{s}(t)=\frac{1}{\sqrt{2 b}}\left(-f_{s}(t)+b \dot{x}_{s}(t)\right)$

Assume delay is $T$, incoming wave variables $u_{s}, v_{m}$ are given as:

$$
\begin{gathered}
u_{s}(t)=\frac{1}{\sqrt{2 b}}\left(f_{s}(t)+b \dot{x}_{s}(t)\right)=u_{m}(t-T) \quad(E q .2) \\
v_{m}(t)=\frac{1}{\sqrt{2 b}}\left(-f_{m}(t)+b \dot{x}_{m}(t)\right)=v_{s}(t-T) \quad(E q .3)
\end{gathered}
$$


Wave Variable Based Teleoperation Method

Passivity can be guaranteed theoretically:

$$
\begin{aligned}
E(t) & =\frac{1}{2} \int_{0}^{t}\left(f_{m}(t) \dot{x}_{m}(t)-f_{s}(t) \dot{x}_{s}(t)\right) d t \\
& =\frac{1}{2} \int_{0}^{t}\left(u_{m}^{T} u_{m}-v_{m}^{T} v_{m}-u_{s}^{T} u_{s}+v_{s}^{T} v_{s}\right) d t \\
& =\frac{1}{2} \int_{t-T}^{t} u_{m}^{T} u_{m} d t+\frac{1}{2} \int_{t-T}^{t} v_{s}^{T} v_{s} d t \geq 0
\end{aligned}
$$

- Any arbitrary time delay caused energy in the transmission will be stored in communication, thus making the system performs passive [H. Ching and W. Book, 2006] 
Wave Variable Based Teleoperation Method Disadvantage:

Good tracking performance is not achieved due to influence of disturbing bias terms:

$$
\begin{aligned}
& f_{m}(t)=f_{s}(t-T)+b\left(\dot{x}_{m}(t)-\dot{x}_{s}(t-T)\right) \\
& \dot{x}_{s}(t)=\dot{x}_{m}(t-T)+\frac{1}{b}\left(f_{m}(t-T)-f_{s}(t)\right)
\end{aligned}
$$


Enhanced Wave Variable Teleoperation Structure

\section{Outline}

(1) Motivation

(2) Enhanced Wave Variable Architecture

- Fundamentals of Wave Variable Teleoperation

- Enhanced Wave Variable Teleoperation Structure

- Effect of Non-Passive Operator on Enhanced Wave Variable Structure

(3) Conclusions and Perspectives 
- It is desired for bilateral teleoperation with time delay to stably get tracking performance as:

$$
f_{m}(t)=f_{s}(t-T) \quad \dot{x}_{s}(t)=\dot{x}_{m}(t-T)(E q .7)
$$

- Enhanced wave variable teleoperation structure [Guo, J., et al, 2015]

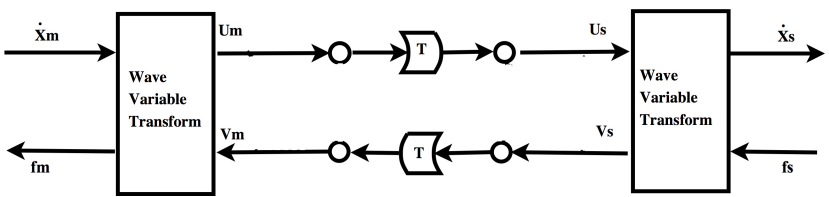

Figure 6: Wave variable teleoperation structure 
- It is desired for bilateral teleoperation with time delay to stably get tracking performance as:

$$
f_{m}(t)=f_{s}(t-T) \quad \dot{x}_{s}(t)=\dot{x}_{m}(t-T)(E q .7)
$$

- Enhanced wave variable teleoperation structure [Guo, J., et al, 2015]

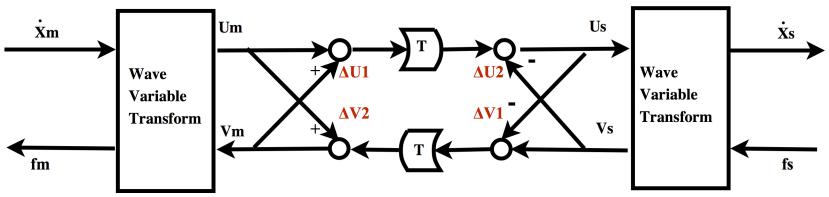

Figure 7 : Enhanced wave variable teleoperation structure

Wave variable compensation terms:

$$
\Delta u=v_{m}(t-T)-v_{s}(t) \quad \Delta v=u_{m}(t)-u_{s}(t-T) \quad(E q .8)
$$


However, wave variable compensation terms may introduce extra energy which destroy the passivity of whole system.

- Energy reservoir based regulators [Munir, S., et al, 2002] Adjusted wave variable compensation terms as:

$$
\begin{aligned}
& \Delta u=\alpha\left[1-e^{-\beta E_{s}(t)}\right]\left(v_{m}(t-T)-v_{s}(t)\right) \quad(E q .9) \\
& \Delta v=\alpha\left[1-e^{-\beta E_{m}(t)}\right]\left(u_{m}(t)-u_{s}(t-T)\right)
\end{aligned}
$$

- $\alpha$ and $\beta$ are positive parameters for tune the regulator;

- $E_{s}(t)$ and $E_{m}(t)$ are energy reservoirs:

$$
\begin{aligned}
& E_{s}(t)=\int_{0}^{t}\left(u_{m}^{2}(t-T)-v_{s}^{2}(t)\right) d t \quad(E q .11) \\
& E_{m}(t)=\int_{0}^{t}\left(v_{s}^{2}(t-T)-u_{m}^{2}(t)\right) d t \quad(E q .12)
\end{aligned}
$$




\section{Outline}

(1) Motivation

(2) Enhanced Wave Variable Architecture

- Fundamentals of Wave Variable Teleoperation

- Enhanced Wave Variable Teleoperation Structure

- Effect of Non-Passive Operator on Enhanced Wave Variable Structure

(3) Conclusions and Perspectives 
- Master, slave, operator and environment are assumed to be passive for aforementioned energy reservoir based regulators.

- Recent research effort indicated that operator is not always passive [Jazayeri, A. et al, 2015]

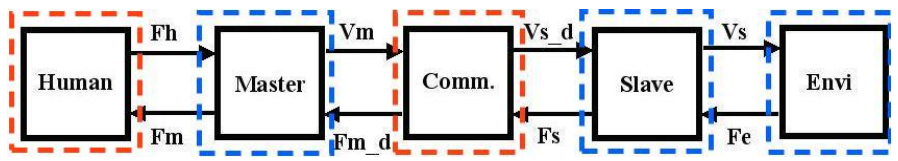

Figure 8 : Non-passive behavior of operator for bilateral teleoperation

- Non-passive behaviors of operator potentially cause extra energy injected into system; 
Recall Eq. 12, with non-passive behaviors of operator, the energy reservoir runs as:

$$
E_{m}(t)=\int_{0}^{t}\left(v_{s}^{2}(t-T)-u_{m}^{2}(t)\right) d t+E_{o} \quad(E q .13)
$$

in which, $E_{o}$ represents the energy injected by the non-passive behavior of operator into system, and might cause Eq.13 to be negative, thus make the wave variable compensation terms be choked off easily. 
Experimental setup:

- Two Omega 7 devices as master and slave separately, a Force sensor (F/T Nano17 Sensor) integrated on slave side;

- A two-layer synthetic phantom was used to mimic the human tissue.

- Time delay was manually set as $200 \mathrm{~ms}$ to quantitatively evaluate the tracking performance.

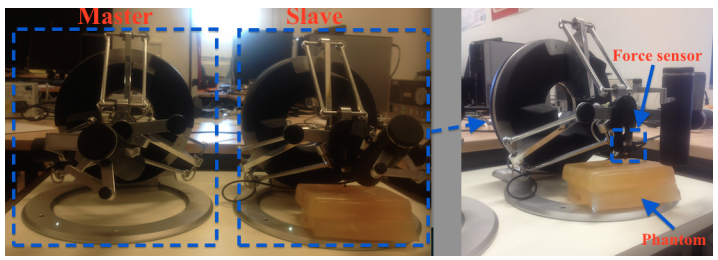

Figure 9 : Experimental setup with two Omega 7 robotic devices 
First case study, record the energy reserved on both sides during possible non-passive operation.

- A 29-year-old male manipulated master (move following sine wave trajectory);

- Safety consideration, energy reservoir were initialized as 500;

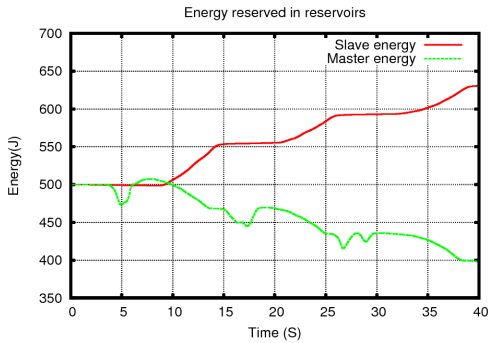

Master side performed non-passive.

Figure 10: Energy reserved in reservoirs on master and slave side with initial energy reservoir as 500 (in contact) 
- Same configuration experiment with lower initialized value of energy reservoir as 50 .

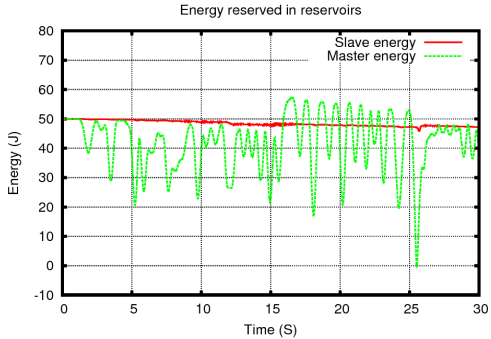

\section{Energy reserved on master side reaches 0 at $26 s$}

Figure 11 : Energy reserved in reservoirs on master and slave side with initial energy reservoir as 50 (free motion) 


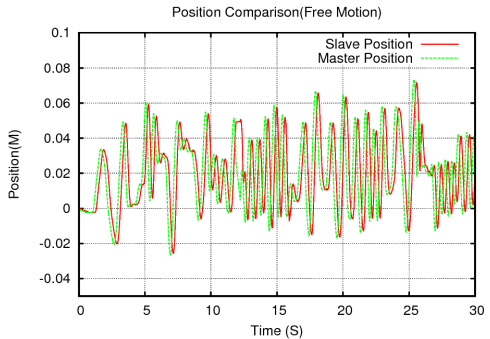

Figure 12 : Position tracking with initial energy reservoir as 50 (free motion)

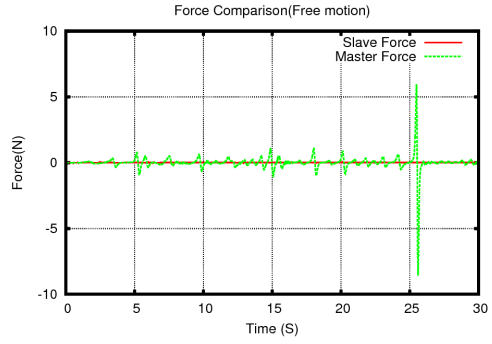

Figure 13 : Force tracking with initial energy reservoir as 50 (free motion) 
- Same configuration experiment with higher initialized value of energy reservoir as 500 .

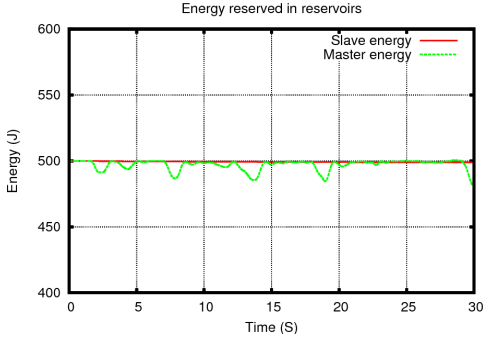

\section{Almost keep passive}

Figure 14 : Energy reserved in reservoirs on master and slave side with initial energy reservoir as 500 (free motion) 


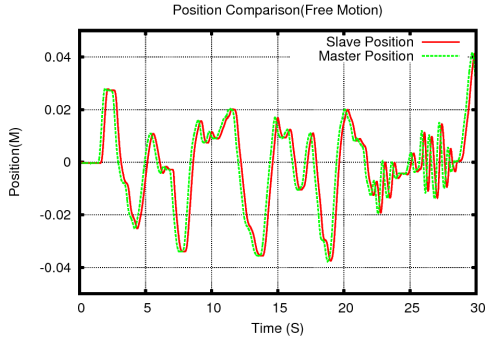

Figure 15 : Position tracking with initial energy reservoir as 500 (free motion)

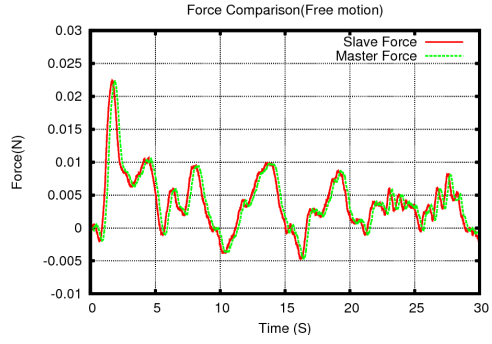

Figure 16 : Force tracking with initial energy reservoir as 500 (free motion) 


\section{Remarks:}

- Non-passive behavior may inject extra energy into bilateral teleoperation system, thus potentially cause stability issues;

- Increase the enegy reservoir initial value can handle occasional non-passive behaviors, but won't work if non-passivity continues over too long; 


\section{Conclusions:}

- Time delay issues in robotic-assisted surgery;

- Enhanced wave variable teleoperation method for improved position and force tracking performance;

- Non-passive behavior of operators may inject extra energy to teleoperation system thus cause stability issue;

- Energy reservoir based regulator can handle occasional non-passive behaviors of operators;

\section{Perspectives:}

- Further theoretical analysis about energy reservoir with non-passive operators;

- Prediction method for further improved force feedback;

- Non-passive environment;

- Variable time delay/data loss issues of communication; 


\section{Thanks for your attention! $Q \& A$.}

